\title{
Laser stripe peak detector for 3D scanners. A FIR filter approach*
}

\author{
Josep Forest, Joaquim Salvi, Enric Cabruja and Carles Pous \\ Universitat de Girona \\ Computer Vision and Robotics Lab. \\ Politècnica P-IV, Campus Montilivi, 17003 Girona, Catalonia \\ \{josep.forest,joaquim.salvi,enric.cabruja,carles.pous\}@udg.es
}

\begin{abstract}
The accuracy of a $3 D$ reconstruction using laser scanners is significantly determined by the detection of the laser stripe. Since the energy pattern of such a stripe corresponds to a Gaussian profile, it makes sense to detect the point of maximum light intensity (or peak) by computing the zerocrossing point of the first derivative of such Gaussian profile. However, because noise is present in every physical process, such as electronic image formation, it is not sensitive to perform the derivative of the image of the stripe in almost any situation, unless a previous filtering stage is done. Considering that stripe scanning is an inherently rowparallel process, every row of a given image must be processed independently in order to compute its corresponding peak position in the row. This paper reports on the use of digital filtering techniques in order to cope with the scanning of different surfaces with different optical properties and different noise levels, leading to the proposal of a more accurate numerical peak detector, even at very low signalto-noise ratios.
\end{abstract}

\section{Introduction}

The reconstruction accuracy depends on a set of crossrelated issues like calibration [8], camera resolution, optics distortion, noise [2,3], etc, while the range acquisition time is dependent on a smart image processing algorithm (responsible of segmenting the appropriate regions of interest) in addition to a fast imaging sensor. A review on laser scanning methods can be found in [5]. In this paper, a numerical peak detector based on the computation of the zero-crossing of the first derivative of each image row is analysed. Similarly to the estimator proposed by Blais and Rioux [1], a derivative filter is used for computing the first derivative,

* This research has been partially funded by the spanish project CYCYT TIC2003-08106-CO2-02. but its coefficients and order are selected according to the surface optical properties and a previous frequency analysis $[7,9,10]$ of the image. The performance in terms of peak location error of this method is similar to that of [1] when the signal-to-noise ratio of the stripe image is high, but it is significantly improved when the nature of the surface or a low light power induce a high noise level in the stripe image [6]. This paper is structured as follows: the next section explains the different surface properties under consideration. The noise sources which affect the 3D measurement are briefly explained in section 3 . Section 4 analyses the proposed method. Section 5 reports on the experimental results and in section 6 a discussion about the method is addressed in the form of conclusions.

\section{Scanning different surfaces}

The optical properties of the surface significantly determine the performance of the laser scanner. The optimal surface type for scanning purposes is a totally lambertian surface with a high reflection index. Figures $1 \mathrm{a}$ and $1 \mathrm{~b}$ show how a light ray behaves under both a specular and a lambertian surface. Translucid surfaces are often present in our everyday life (certain types of plastic, animal tissue, silicon, resins, certain rocks or minerals, etc.). Figure 1c shows how a ray of light behaves when it impinges such kind of surface. In a translucid surface, light reflects as in a lambertian surface, but it goes through the material until a certain depth. The higher the light power, the deeper the light penetrates inside the material. In addition, the light scatters inside the material, so that a camera looking at it "sees" laser reflexions sourcing from inside it. See [6] for an example of light behaviour and a study of how it affects the 3D measurement on marble surfaces. Figure 2 (right), shows a laser stripe reflected on a lambertian surface, while figure 2 (left), shows how the reflection on a translucid surface is seen by the camera. As it is shown, a laser stripe impinging on a translucid surface induces a lot of undesired light peaks where they are not expected to be. In addition, 


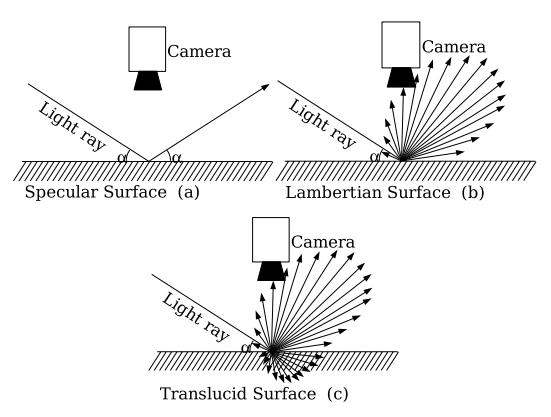

Figure 1. Behaviour of light reflected on a specular surface (a), a lambertian surface (b) and a translucid surface (c).

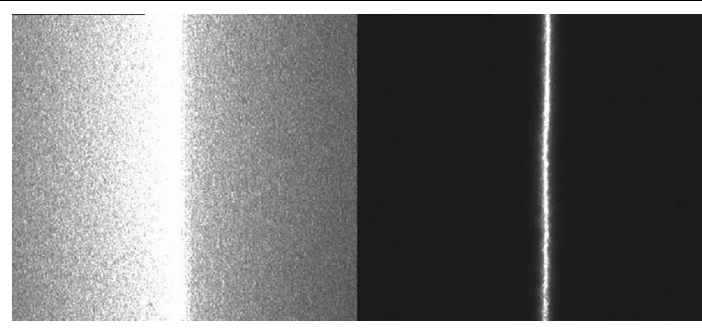

Figure 2. A laser stripe on a translucid (left) and a lambertian (right) surface.

if the light power is lowered, the noise due to the different sources becomes more and more significant and hence, the reconstruction quality degrades.

\section{Noise sources}

In standard cameras, the most influencing noise has been found to follow a Gaussian probability distribution, which is a consequence of the point spread function, due to the imperfections in the lenses and the grey level digitisation. Three noise sources have been found to influence the threedimensional measurement of camera-laser based 3D scanners: electrical noise, quantisation noise and speckle. The latter noise source is directly related to the nature of laser light, while the former two are inherent to the image sensor. Electrical and quantisation noise are very significant when the $\mathrm{S} / \mathrm{N}^{1}$ is very low, i.e. when the stripe light power is very low. A thorough study about the performance of CMOS image sensors and how electrical noise affects the image quality can be found in [3]. This is commonly found when the

1 S/N: Signal-to-noise ratio surface exhibits a very low reflection index or when scanning at high speeds. In addition, speckle noise influences dramatically on the measurement. Speckle is due to the reduced wavelength of light compared to the surface roughness and the monochromacity of laser light [2] and influences the 3D measurement. These three noise sources, combine together and make the observer see the constructive and destructive interferences within the laser stripe.

\section{Filtering and detection}

Either due to any of the noise sources explained in the previous section or the scattering of light inside a translucid material, the camera images a laser stripe with a certain amount of undesired lighting peaks superimposed to it. From the point of view of signal processing, it seems reasonable to consider it as the manifestation of a noisy signal, which complies with the principle of superposition. Regarding each row of the stripe image as a signal, a digital low pass filter can be designed with the right cut-off frequency, attenuation and transition band width parametres. There are several methods for obtaining the filter coefficients. Haddad et.al. [7] developed a new technique for designing FIR $^{2}$ filters based on the method of vector-space projection. Other approaches include the method of adjustable windows [9] or the use of genetic algorithms for certain types of FIR filters [10]. Overall, the estimators presented in the litterature are very sensitive to the variations in $\mathrm{S} / \mathrm{N}$, seriously constraining their performance on many types of materials, especially considering translucid surfaces. In this work, the computiation of the zero crossing point of the first derivative of each stripe image row has been considered for obtaining an estimation of the peak position. Although other methods use the same approach, in the present work much more emphasis has been put in the obtention of the derivative operator, generalising the notation in terms of the convolution operation. As stated in equation 1 FIR filtering consists on computing the convolution of the row signals $(x(n))$ with the coefficients of the filter $h(n)$. As it has been shown, the first derivative of the convolution of $x(n)$ and the filter coefficients $h(n)$ is equal to the convolution of $x(n)$ and the first derivative of $h(n)$. Once the filtered first derivative has been computed, assuming that the $\mathrm{S} / \mathrm{N}$ is high enough for differentiating the laser stripe from the rest of the scene by simple thresholding, the method for obtaining the zero crossing point is as follows. Formerly, the maximum grey level value is selected. Second, the signal is tracked from its maximum, left to right in the image, until the first negative value is found. Finally a straight line is computed between the points corresponding to the first negative and the last positive signal values, and the zero crossing is computed. Figure

2 FIR: Finite Impulse Response 


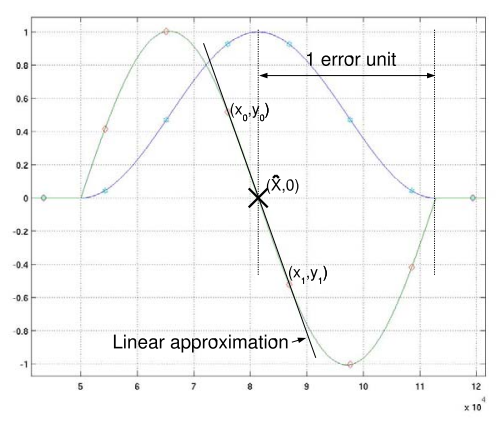

Figure 3. Laser peak and first derivative.

3 summarises this process and equation 3 shows how the estimation of the zero crossing is computed.

$$
y(n)=\sum_{k=0}^{M} x(k) \cdot h(n-k) \triangleq x(n) * h(n)
$$

The first derivative of the filtered row, $\frac{d y}{d n}$, can be computed as shown in equation 2 , according to the properties of convolution.

$$
\begin{aligned}
& \frac{d y(n)}{d n}=\frac{d[x(n) * h(n)]}{d n}=\frac{d \sum_{k=0}^{M} x(k) \cdot h(n-k)}{d n}= \\
& =\sum_{k=0}^{M} \frac{d[x(k) \cdot h(n-k)]}{d n}=\sum_{k=0}^{M} \frac{d x(k)}{d n} \cdot h(n-k)+ \\
& +\sum_{k=0}^{M} x(k) \cdot \frac{d h(n-k)}{d n}=\sum_{k=0}^{M} x(k) \cdot \frac{d h(n-k)}{d n}= \\
& =x(n) * \frac{d h(n-k)}{d n} \\
& \hat{X}=x_{0}-\frac{y_{0} \cdot\left(x_{1}-x_{0}\right)}{y_{1}-y_{0}}
\end{aligned}
$$

\section{Results}

In order to evaluate the effect on the peak estimation, two experiments have been arranged. The former consists on evaluating the behaviour of the proposed method on a mate surface, for different $\mathrm{S} / \mathrm{N}$ values, comparing with 5 other peak estimators. The performance of these peak estimators has been previously studied by [4], and are known as Gaussan approximation (GA), Centre of mass (CM), Linear approximation (LA), Blais and Rioux detector $(\mathbf{B R})$ and Parabolic estimator (PA). The description of these methods falls beyond the scope of this paper, however, table 3 enumerates their mathematical equations. The BR detector, uses a signal $g(n)$ for estimating the peak position. This $g(n)$ is the filtered and derived row signals, using the filter proposed in the Blais and Rioux work [1].

In the second experiment, a piece of translucid white plastic has been partially painted in a mate, white colour. The thickness of the paint layer is $60 \mu \mathrm{m}$ in average. This experiment is similar to that performed by [6]. The filter coefficients have been previously estimated using the Matlab $f d a$ tool function.

In the first experiment, the image with the lowest $\mathrm{S} / \mathrm{N}$ has been chosen for computing the filter coefficients. In the second case the most noisy side of the image is the one corresponding to the response of a translucid surface, hence this side of the image has been used for obtaining the filter coefficients. In both cases, the stop-band has been chosen to have an attenuation of $80 \mathrm{~dB}$. The cut-off frequency and the width of the transition band have been selected after the study of the row signals in the frequency domain. In the first experiment, the filter order has been 56 while for the second experiment the filter order has been selected to be 296 . These results are consistent with the fact that the laser impinging on the translucid surface show a much higher noise level, and that the stripe is significantly wider in the second case than in the first one.

In the first experiment, the 6 peak estimators have been applied to the whole stripe image, computing the standard deviation of the peaks on each image, for $4 \mathrm{~S} / \mathrm{N}$ levels. This results are summarised in table 1 . In this table, the Proposed Method has been identified with the acronym "PM". As can be observed, PM keeps a lower and more constant value of $\sigma$ compared to the other methods for any $\mathrm{S} / \mathrm{N}$ value, even when it is considerably low.

In the second experiment, the stripe is considerably wider than in the former one, with a large saturation width (i.e. each row peak is far from fitting a pure Gaussian shape). This prevented us to use the 6 methods, since GA, LA and $\mathrm{PE}$ can not be applied without modifications in the algorithms. Instead, we have used only PM, BR and CM algorithms, computing the standard deviation in both sides -opaque and translucid- of the surface for two S/N conditions. It is worth noting that speckle noise is much more significant under these contitions than thermal noise. This makes the S/N levels to be lower for high light power than low light power. In addition, considering the peak estimation on a translucid material, this effect is magnified due to the light scattering. Furthermore, a bias is observed when estimating the peak on the translucid material. Table 2 compiles the peak estimation data in terms of standard deviation and mean values. It is worth observing that for opaque surfaces, PM shows better results (in terms of $\sigma$ ) for low light power than for high power. This is due to the wider image of the stripe for high light power. The peak estimation for translucid surfaces, however, is better for high than low light power. CM is the best option under these conditions, but it has been shown previously that $\mathrm{CM}$ is not a good choice when the stripe is narrow. Comparing PM and $\mathrm{BR}$, using filters of the same order but different coefficients, PM performs better in both light power conditions and both 


\begin{tabular}{c|cccc}
\hline SN $\triangleright$ & $13.34 \mathrm{~dB}$ & $8.12 \mathrm{~dB}$ & $4.13 \mathrm{~dB}$ & $0.92 \mathrm{~dB}$ \\
\hline \hline $\mathrm{PM}$ & 1.719 & 1.712 & 1.728 & 1.777 \\
$\mathrm{BR}$ & 1.755 & 1.799 & 1.910 & 4.228 \\
$\mathrm{CM}$ & 1.744 & 1.809 & 1.813 & 1.892 \\
$\mathrm{GA}$ & 1.759 & 1.842 & 1.816 & 1.910 \\
$\mathrm{LA}$ & 2.194 & 3.088 & 2.159 & 1.890 \\
$\mathrm{PE}$ & 1.792 & 1.833 & 1.820 & 1.889
\end{tabular}

Table 1. Values of $\sigma$ estimating the peak using the 6 methods with $4 \mathrm{~S} / \mathrm{N}$ levels.

\begin{tabular}{|c|c|c|c|c|c|}
\hline \multirow{2}{*}{$\begin{array}{c}\triangle \\
\text { Light }\end{array}$} & \multicolumn{2}{|c|}{ Opaque (11dB) } & \multicolumn{3}{|c|}{ Trans. $(7 \mathrm{~dB})$} \\
\hline & $\bar{X}$ & $\bar{\sigma}$ & $\bar{X}$ & $\bar{\sigma}$ & $\triangle \hat{X}$ \\
\hline$\overline{\overline{\mathrm{PM}}}$ & (382.841 & $\overline{1.862}$ & 338.361 & $\overline{44.258}$ & 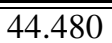 \\
\hline BR & 0.719 & 2.314 & 321.756 & 4.560 & 58.963 \\
\hline $\mathrm{CM}$ & 378.858 & 1.895 & 350.054 & 2.132 & 28.804 \\
\hline$\nabla$ & \multicolumn{2}{|c|}{ Opaque (21dB) } & \multicolumn{3}{|c|}{ Trans. $(12 \mathrm{~dB})$} \\
\hline Light & $\bar{X}$ & $\sigma$ & $\bar{X}$ & $\sigma$ & $\triangle \bar{X}$ \\
\hline$\overline{\overline{\mathrm{PM}}}$ & 3.647 & 1.639 & $\overline{365.478}$ & $\overline{\overline{6.010}}$ & $\bar{~} 18.168$ \\
\hline $3 \mathrm{~K}$ & & 1.640 & 363. & 9.363 & 19.501 \\
\hline $\mathrm{CM}$ & 379.732 & 1.519 & 355.054 & 2.687 & 24.677 \\
\hline
\end{tabular}

Table 2. Mean value and $\sigma$, estimating the peak on two types of material under different light power conditions ( $\mathrm{S} / \mathrm{N}$ values in $\mathrm{dB}$ ).

types of materials, especially with low $\mathrm{S} / \mathrm{N}$. In addition, the logical bias in the measurement of the translucid material ( $60 \mu \mathrm{m}$ of paint thickness) varies in both light power situations, and is more significant using BR than PM, as stated in the column labelled $\triangle \hat{X}$ in table 2 . PM and BR are much more sensitive to light changes than $\mathrm{CM}$, however, a visual inspection let us observe that $\Delta \hat{X}$ is closer to reality under low light power and better estimated by PM and BR.

\section{Conclusions and further work}

A new method for estimating the peak position of a laser stripe has been reported, and its performance has been compared with other existing 5 methods. The experimental results show that the proposed method yield to better estimations of the peak position, especially when the $\mathrm{S} / \mathrm{N}$ is very low. When translucid surfaces are scanned, a bias in the peak estimation appears, which is a function of the impinging light power. The computational complexity of the proposed method is similar to that of BR but increases its accuracy in a wider range of stripe light power or noncooperative surfaces. The strong point of this method is the analysis of the row signals in the frequency domain, which

\begin{tabular}{l|l}
\hline Estim. & Formulae \\
\hline \hline BR & $\hat{\delta}= \begin{cases}\frac{g(i)}{g(i)-g(i+1)} & f(i+1)>f(i-1) \\
\frac{g(i-1)}{g(i-1)-g(i)} & f(i+1)<f(i-1)\end{cases}$ \\
GA & $\hat{\delta}=\frac{1}{2} \cdot\left(\frac{\ln (a)-\ln (c)}{\ln (a)+\ln (c)-2 \cdot \ln (b)}\right)$ \\
CM & $\hat{\delta}=\frac{c-a}{a+b+c}$ \\
LA & $\hat{X}= \begin{cases}x-\frac{a-c}{2(b-a)} & c>a \\
x-\frac{a-c}{2(b-c)} & \text { otherwise }\end{cases}$ \\
PA & $\hat{\delta}=\frac{1}{2} \cdot \frac{a-b}{c-2 \cdot b+a}$ \\
\hline
\end{tabular}

Table 3. Estimator formulae. The $\hat{\delta}$ stand for the subpixel offset. The $a, b$ and $c$ stand for the 3 consecutive pixels of the peak, where $b$ is the maximum in intensity value.

yields to the cut-off frequency and the transition band width for obtaining the coefficients of an optimised filter.

\section{References}

[1] F. Blais and M. Rioux. Real-time numerical peak detector. In Signal Processing, volume 11, pages 145-155, 1986.

[2] B. L. Curless. New methods for surface reconstruction from range images. PhD thesis, Stanford University, 1997.

[3] B. Dierickx. Cmos image sensors: concepts and limits. A short course given at Photonics West 2000, January 2000.

[4] R. Fisher and D. Naidu. A comparison of algorithms for subpixel peak detection. In Proc. 1991 British Machine Vision Association Conf, pages 217-225, 1991.

[5] J. Forest and J. Salvi. A review of laser scanning threedimensional digitisers. In Intelligent Robots and Systems, 2002, pages 73-78. IEEE/RSJ, 2002.

[6] G. Godin, M. Rioux, J. A. Beraldin, M. Levoy, L. Cournoyer, and F. Blais. An assessment of laser range measurement on marble surfaces. In 5th Conference on Optical 3D Measurement Techniques, pages 49-56, October 2001.

[7] K. C. Haddad, H. Stark, and N. P. Galatsanos. Constrained fir filter design by the method of vector space projections. IEEE Transactions on circuits and systems II: Analog and digital signal processing, 47(8):714-725, august 2000.

[8] J. Salvi, X. Armangué, and J. Batlle. A comparative review of camera calibrating methods with accuracy evaluation. Pattern Recognition, 35:1617-1635, August 2002.

[9] T. Saramaki. Adjustable windows for the design of fir filters - a tutorial. In Proceedings of the 6th Mediterranean Electrotechnical Conference, pages 28-33, May 1991.

[10] D. Suckley. Genetic algorithm in the design of fir filters. In Circuits, Devices and Systems, IEE Proceedings G, volume 138, pages 234-238, April 1991. 